\title{
Magnetic Resonance Imaging of the Abdomen and Pelvis without Contrast
}

National Cancer Institute

\section{Source}

National Cancer Institute. Magnetic Resonance Imaging of the Abdomen and Pelvis

without Contrast. NCI Thesaurus. Code C137873.

Magnetic resonance imaging of the abdomen and pelvis, without the use of a contrast agent. 